\title{
Application of Large Scale MIMO Technology in the Heterogeneous Networks
}

\author{
Zhiyong Ren \\ School of Communication Engineering, \\ ChongQing College of Electronic Engineering, ChongQing 401331, China \\ E-mail: renzhiyongtxxy@sina.com
}

\begin{abstract}
To deploy the MIMO technology in the heterogeneous networks, we analyzed the interference and energy efficiency of the system. The recent studies of the application of the MIMO technology in heterogeneous network is introduced first. Then, The system model, inter cell interference are analyzed. The mathematical model was proposed in the paper involving energy efficiency. Finally, the simulation results in the study indicates that the simple prevention can eliminate the interference and the ZF pre-encoding is equal to the MRT pre-encoding under limited conditions. The results show that the interference model and energy efficiency equation proposed in the study have a great application prospect.
\end{abstract}

Keywords: MIMO, heterogeneous network, energy efficiency analysis.

\section{Introduction}

In the past few years, Multiple input multiple output (MIMO) technology, along with the doubled mobile data transmission volume and the rapid increase in the use of smart phones and new mobile terminal devices, as well as the rapid development of mobile Internet. During the recent two years, the intelligent mobile phone usage has increased three times than before, while the mobile data traffic has increased 2.3 times [1]. The industry generally believes that the future of this growth will continue in the future. The amount of mobile data has increased 24 times from 2010 to 2015, and it still will increase 500 times from 2010 to 2020. The survey found that the $70 \%$ to $80 \%$ mobile data traffic is generated by the indoor hot spots, therefore the indoor signal coverage has been the top priority in the mobile network optimization. Due to the situation of concentrate user distribution, buildings' electromagnetic wave shielding and absorption effect, radio wave transmitted from base station has declined dramatically, thus the signal strength is very weak in indoor environment. Based on the situation described above, how to realize the high speed wireless communication in indoor environment has become a difficult problem in the industry.

This paper proposed a network deployment of the future mobile communication network, that is, the micro cellular deployment of heterogeneous networks. Macro base station is for user communication coverage with low bit rate requirements, and the micro base station is for hot users within a small range of communication services. Such heterogeneous network deployment not only can improve the mobile access rate but also reduce the total power of the system. The increase of the density of the micro cell can reduce the cell coverage radius and the power consumption of transmission loss, while maintain the high number of service users in a single micro cell and improve the system frequency to achieve broadband high-speed communications. To this basis, the heterogeneous network of LTE-A is an important 
candidate for the network deployment in the next generation mobile communication system.

A study[2] give the application analysis of the large scale MIMO in the TDD in heterogeneous network, the study points out that the base station's configuration of large scale antenna array, and the micro base station structure using the conventional multi antenna system, at the same time, the micro area uses time division duplex channel deployment. And another study gives another kind of heterogeneous network model, which separates the macro and the micro cellular frequency bands. Macro base station still adopts the present $2 \mathrm{GHz}$ band, while the micro base station using $3 \mathrm{GHz}$ or even higher frequencies. The inventor of the MIMO technology Marzetta [3] has pointed out that the future mobile communication may be in the range of $10 \mathrm{GHz}$ band. In the indoor enclosed corridor environment, the research on the time and spatial characteristics of the broadband wireless channel in $14 \mathrm{GHz}$ band has been studied [4]. Channel parameters of the high frequency channel are analyzed by means of channel modeling, including path loss, shadow fading, delay spread and angle spread. The conclusion shows that the time delay spread in the high frequency band is minimal, and the LOS (line of sight) environment can increase the coverage of the electromagnetic wave in the NLOS (non-line of sight) environment. These results indicate that the high frequency segment has great potential in the field of wireless communication.

In view of the research on the contents of the heterogeneous networks and high frequency communication, the application of large scale MIMO technology in heterogeneous networks is given by a study [5]. Assume that macro station is still using the traditional multi antenna and micro base station is configured of large scale antenna array. In such a heterogeneous network deployment system, a large scale antenna array of micro base stations is working at high frequency. Since the loss of high frequency signal transmission, it is easy to reduce the inter cell interference in the high frequency signal transmission, and the large antenna array is relatively easy to implement in high frequency communication environment [6]. The heterogeneous network system not only realize the high frequency spectrum efficiency and low emission power of large-scale MIMO system, but also can inhibit the interference between the cells, which provides the possibility of the future mobile communication[7]. While taking into account the needs of indoor communications, it can be installed in the interior of the home base station to achieve a seamless coverage of local high speed transmission.

Both transceiver requirement of communication system in MIMO uses multiple antennas for communication. After more than 20 years of development, the MIMO technology has been developed from single user MIMO technology to multi user MIMO technology [8]. As early as 1990s, the Bell Laboratory establish the first MIMO experimental system, and measured 20bit/(s* Hz) spectral efficiency [9], it creates a groundbreaking work of MIMO. With the development of MIMO technology, we can use the space diversity technology to improve the quality of the link, and improve the system throughput without increasing the system spectrum resource.

Researches have been compared of the channel capacity of MIMO system in the last century [10]. In the ideal condition, the channel capacity increases linearly with the number of transmit antennas. In the actual system, the MIMO system is difficult to reach its theoretical value due to the limitation of the correlation between the antenna and the surrounding environment [11]. And in the cellular communication environment, the MIMO is expected to increase the system throughput in the same time and frequency resources. 


\section{System Model}

Home base station is also known as the Femtocell, it is a low power, low cost, easy to operate, easy configuration, self-installed small mobile cellular communications base station [12]. Femtocell is mainly for home networks, enterprises, universities and other public hotspots of small communication coverage. Home base station can be connected with the user's wired broadband network, without the need to go through the cellular network, and it can meet the needs of a certain rate of communication [13]. Assuming that the base station is working in the $10 \mathrm{GHz}$ frequency band, the communication between the macro and micro cellular base station is not interfered.

Micro base station, according to the communication demands, distributed in the macro cellular network, the cell radius is about $100 \mathrm{~m}$ to $1000 \mathrm{~m}$, the transmission power is relatively low, about 20 to $30 \mathrm{dBm}$. It is assumed that the high frequency section of the micro base station is separated from the working frequency band of the macro cellular which is $3 \mathrm{GHz}$, and the base station antenna is configured for a large array [14]. The system uses time division duplex communication mode.

Macro base station is the earliest cellular mobile communication technology, its coverage is relatively large, the cell radius is more than $10 \mathrm{~km}$; transmission power is also relatively high in $46 \mathrm{dBm}$ above. The macro cell using traditional multi antenna technology, and still work in the current $2 \mathrm{GHz}$ band, Macro station also uses time division duplex communication mode.

To build a heterogeneous network, the principles are as follows: in accordance with the needs of the user to deploy the outage rate of micro cell base station and other facilities [15]. Interrupt rate refers to the percentage of users in a certain area has lower downlink than a preset number of all users within a cell comparing to the whole population. In some areas, the interrupt rate of heterogeneous network is higher than the system provided, according to the high rate of the interruption of the heterogeneity network in the hot spots, Femtocell or macro cell are needed for deploying [16]. This is composed by the macro cell, small area and the home base station heterogeneous network, the model of heterogeneous networks is showed in figure 1 .

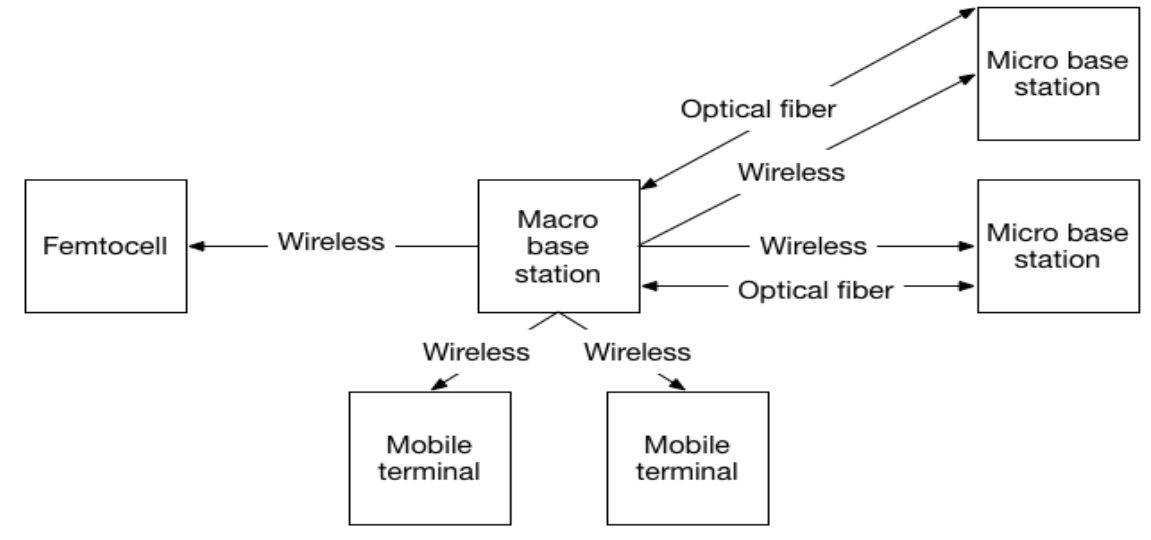

Figure 1. The Structure Model of a Heterogeneous Network

Figure 1 describes a heterogeneous network structure model including macro base station, micro base station, and home base station. In order to reduce the inter cell interference in the heterogeneous network, we use the frequency band separation mode. Macro cellular works in $2 \mathrm{GHz}$ band, the band has the largest transmitter power and the coverage area. The micro cellular network distributes in the hot spot and blind spots, its working frequency is higher than $3 \mathrm{GHz}$, and the communication radius is about several hundred meters, while the transmission power is relatively 
low. Home base station is connected with the cable broadband, the coverage area and the radio frequency power is the lowest, the working band at around $10 \mathrm{GHz}$.

\section{Multi cell MIMO Technology}

\subsection{The MIMO System Model}

The downlink and uplink model of multi cell MIMO system is shown in figure 2. The system has $L$ cells, each base station is equipped with $M$ antennas while serving $K$ single antenna users. As with the single cell, the number of users per cell is unchanged, the number of base station antennas $M$ is infinite. According to the reciprocity of the channel, from the uplink channel matrix $G_{n l}=H_{n l} D^{1 / 2}=\left[g_{n l 1}, \ldots, g_{n l k}\right], g_{n l k}=\left[g_{n l l k}, \ldots, g_{n l M k}\right]^{T},(k=1, \ldots, K)$, the downlink channel matrix is $G_{l}^{H}=D^{1 / 2} H_{l}^{H} . H_{l}$ Is the fast fading matrix of the user $n$ to the base station $l$ in the cell.

So, from the $k$ user of cell $n$ to $m$ antennas of cell $l$, the channel gain is $g_{n l}=h_{n l} \sqrt{\beta_{n l}}$.

Then, the $K$ users in cell ${ }^{n}$ has the receiving signal vector:

$$
y_{n}=\sqrt{p} \sum_{l=1}^{L} G_{n l}^{H} F_{l} x_{l}+n_{n}
$$

In (1), $y_{n}=\left[y_{n 1}, \ldots, y_{n K}\right]^{T}$ is the receiving signal vector of $K$ users in cell $n$, $x_{l}=\left[x_{l 1}, \ldots, x_{l K}\right]^{T}$ is the sending signal vector and $E\left\{\left\|x_{l}^{2}\right\|\right\}=1 . P$ is the the transmission power of each base station, $G_{n l}^{H}$ is the channel gain matrix from $l$ base station to cell $n$.

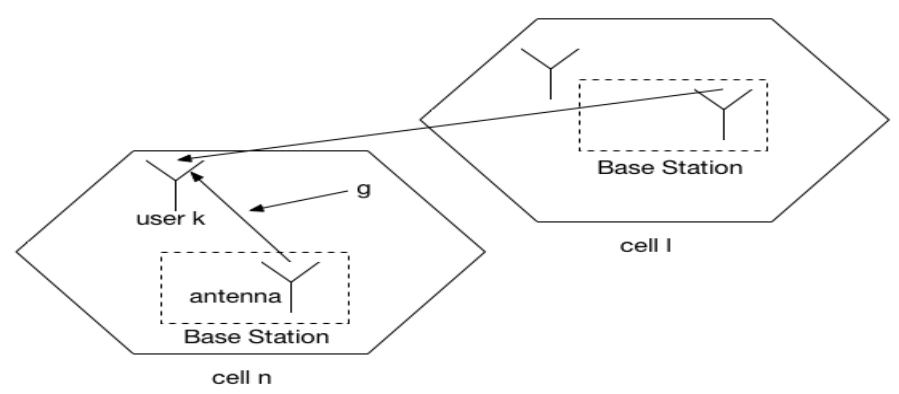

Figure 2. Model of Multi Cell MIMO System

\subsection{Duplex Mode}

Each base station uses TDD mode, because the TDD mode has a low delay comparing to the FDD mode, and it can communicate in the asymmetric frequency, has the advantages of asymmetric transmission of uplink and downlink rate ${ }^{[17]}$. These advantages are very beneficial to the resources allocation of heterogeneous networks, and also conducive to the channel estimation of the large scale MIMO system for the micro cell base station. Figure 3 shows three kinds of TDD working pattern of heterogeneous networks.

Figure 3-(a) is the simplest TDD method for heterogeneous networks. It works in the same frequency band, such as the macro cellular and micro cellular interference. In order to reduce the interference, we general use the power control, cooperative communication and other technologies. Because the working frequency of the home base station is separated from the macro and micro base station, it can effectively avoid the same frequency interference in the same macro cell. 
Figure 3-(b) model is proposed by another study, which is based on macro, micro cellular and the same frequency improved TDD model. The system will stagger the duplex macro cell and microcell, use the macro cell for uplink communication and micro cellular for downlink communication. The purpose of this is to reduce the interference between macro and micro cell edge users and the base station, but cannot avoid the same frequency interference between the base stations.

Figure 3-(c) model is proposed in another study. The method is based on frequency separation. Because the working frequency of the macro and micro cellular is separated, the interference of the cell edge users can be suppressed effectively. At the same time, the MIMO system can meet the requirements of highspeed, stable quality of service (QoS) in high standard mobile communication user.
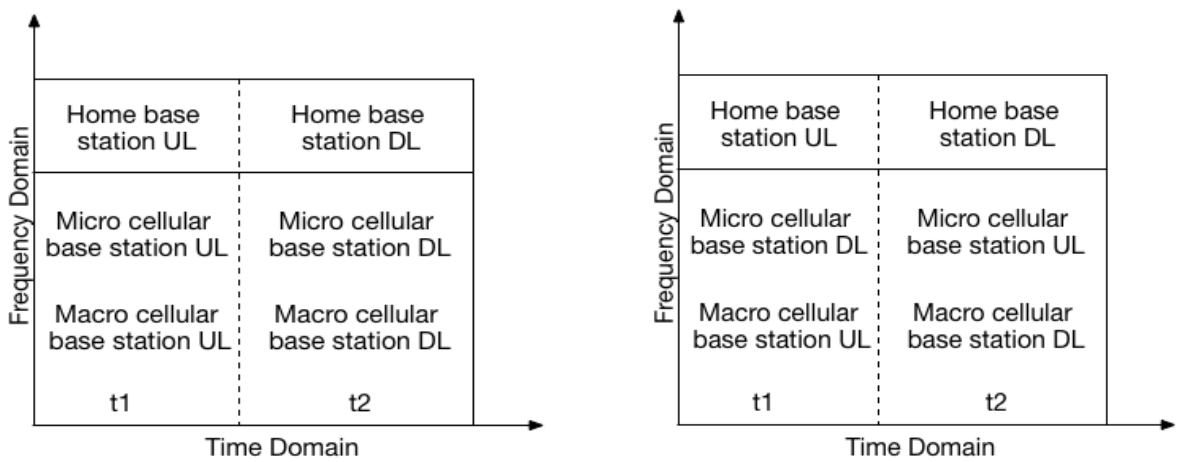

(a) Co-channel TDD

(b) Improved Same Frequency Co-channel TDD

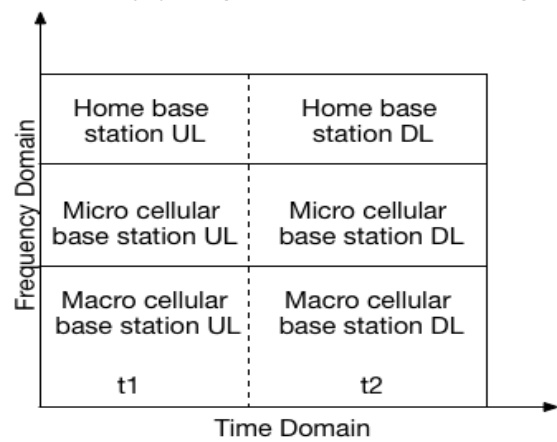

(c) Band separation TDD

Figure 3. Duplex Methods in Heterogeneous Network

\subsection{Inter Cell Interference Analysis}

Figure 4 shows the three duplex model of inter cell interference. In figure 3 , the real line represents the uplink or downlink communication, the dotted line represents the interference. The assumption is that the transmission power of the home base station and the penetration ability of the high frequency segment will not affect the communication of macro or micro users. Figure 4-(a) is the simplest heterogeneous network communication model, and the interference can't be avoided. For figure 4-(b), although the system macro and micro cell are separated by the duplex mode, the interferences still exist. Figure 4-(c) does not exist the same frequency interference, which is based on the exchange of high frequency band for the interference suppression and improved the spectrum efficiency.

Figure 4 shows that the system interference is divided into following types. The first is the uplink communication interference of macro base station and macro cell user to micro base station uplink communication interference. The second is the downlink communication interference when macro cell user receives the macro base 
station signals, other downlink communication interference includes the micro cell user receiving signals from the macro base station.

Figure 4-(c) adopted the communication scheme of frequency band separation, and it can effectively restrain the inter cell interference. Comparing the three schemes in figure 3, the third scheme proposed by this paper not only can effectively suppress the inter cell interference, but also can greatly improve the communication rate and quality of the heterogeneous network.

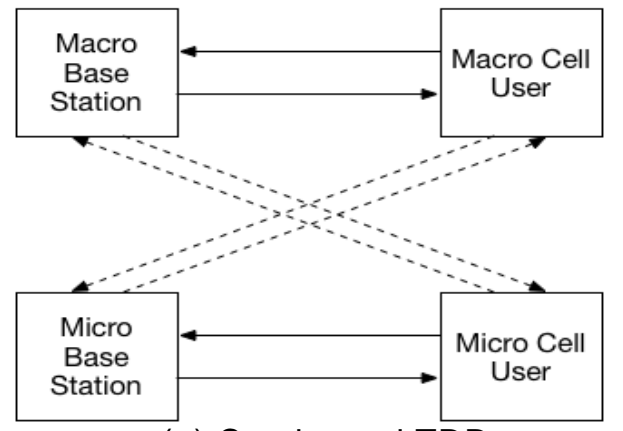

(a) Co-channel TDD

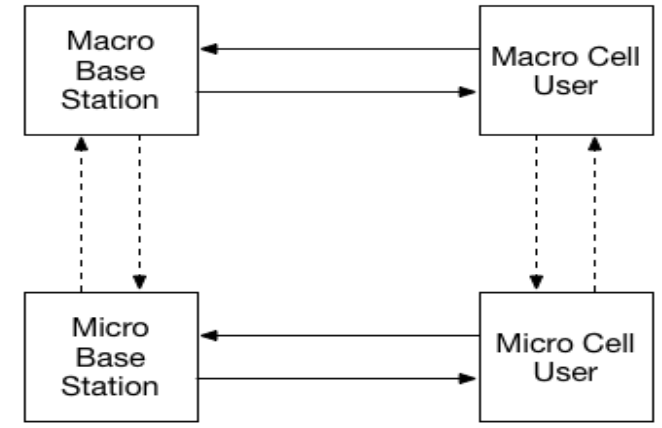

(b) Improved co-channel TDD
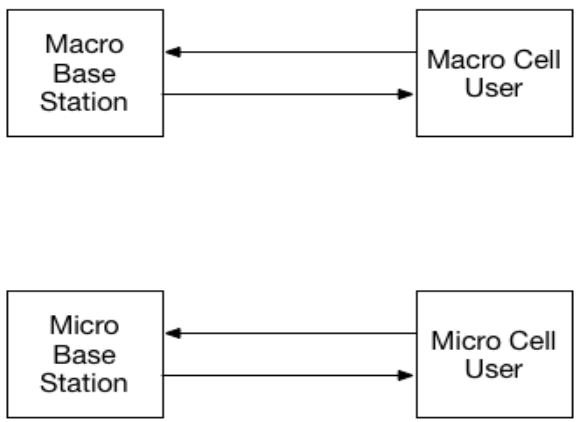

(c) Frequency Separated TDD

\section{Figure 4. Three Inter Cell Interference Model}

\subsection{Performance Analysis}

Same as the single cell MIMO system, we analyzed the spectrum efficiency of the system when the single antenna base station transmit power was reduced to $1 / M$. Under the condition of imperfect channel state information, we also analyzed the relationship between the system throughput and the transmission frequency of the transmitted sequence. We also considered two pre-encoding scheme of MRT, and assumed no correlation between antennas.

In multi cell MIMO system, the $k$ user's received signal of cell $n$ is:

$$
y_{n k}=\sqrt{P} g_{n k}^{H} f_{n k} x_{n k}+\sqrt{P} \sum_{i=1}^{k} g_{n i}^{H} f_{n i} x_{n i}+\sqrt{P} \sum_{l=1}^{l} \sum_{i=1}^{k} g_{n l k}^{H} f_{l i} x_{n k}
$$

In (2), on the right side of the equation, the first term is the useful signal, second term is intra cell interference signal, third term is inter cell interference signal. Thus the spectrum efficiency of cell $n$ is:

$$
\sum_{k=1}^{K} R_{n k}=\sum_{k=1}^{K} E\left\{\log _{2}\left(1+\frac{\frac{P}{K}\left|g_{n n k}^{H} f_{n k}\right|^{2}}{\frac{P}{K} \sum_{i=1}^{K}\left|g_{n n k}^{H} f_{n i}\right|^{2}+\frac{P}{K} \sum_{l=1}^{L} \sum_{i=1}^{K}\left|g_{\ln k}^{H} f_{l i}\right|^{2}+1}\right)\right\}
$$




\subsection{Energy Efficiency Analysis}

The spectrum efficiency of the multi cell MIMO system with known CSI and finite CSI are:

$$
\begin{aligned}
R_{M, P}^{A} & =\sum_{k=1}^{K} E\left\{\log _{2}\left(1+\frac{\frac{P}{K}\left|g_{n n k}^{H} f_{P, n k}^{A}\right|^{2}}{\frac{P}{K} \sum_{i=1}^{K}\left|g_{n n k}^{H} f_{P, n i}^{A}\right|^{2}+\frac{P}{K} \sum_{l=1}^{L} \sum_{i=1}^{K}\left|g_{\ln k}^{H} f_{P, l i}^{A}\right|^{2}+1}\right)\right\} \\
R_{M, I P}^{A} & =\sum_{k=1}^{K} E\left\{\log _{2}\left(1+\frac{\frac{P}{K}\left|g_{n n k}^{H} f_{I P, n k}^{A}\right|^{2}}{\frac{P}{K} \sum_{i=1}^{K}\left|g_{n n k}^{H} f_{I P, n i}^{A}\right|^{2}+\frac{P}{K} \sum_{l=1}^{L} \sum_{i=1}^{K}\left|g_{\ln k}^{H} f_{I P, l i}^{A}\right|^{2}+1}\right)\right\}
\end{aligned}
$$

Assume $P_{u}=\frac{P}{K}$ is the transmit power for every user, from (4) and (5) the energy efficiency are:

$$
\begin{gathered}
\eta_{M, P}^{A}=\frac{1}{P_{u}} \sum_{k=1}^{K} E\left\{\log _{2}\left(1+\frac{\left|g_{n n k}^{H} f_{P, n k}^{A}\right|^{2}}{\sum_{i=1}^{K}\left|g_{n n k}^{H} f_{P, n i}^{A}\right|^{2}+\sum_{l=1}^{L} \sum_{i=1}^{K}\left|g_{\ln k}^{H} f_{P, l i}^{A}\right|^{2}+\frac{1}{P_{u}}}\right)\right\} \\
\eta_{M, I P}^{A}=\frac{(T-\tau)}{T P_{u}} \sum_{k=1}^{K} E\left\{\log _{2}\left(1+\frac{\left|g_{n n k}^{H} f_{I P, n k}^{A}\right|^{2}}{\sum_{i=1}^{K}\left|g_{n n k}^{H} f_{I P, n i}^{A}\right|^{2}+\sum_{l=1}^{L} \sum_{i=1}^{K}\left|g_{\ln k}^{H} f_{I P, l i}^{A}\right|^{2}+\frac{1}{P_{u}}}\right)\right\}
\end{gathered}
$$

In limit conditions, when $P_{u} \rightarrow 0$ :

$$
\begin{aligned}
\lim _{P_{u} \rightarrow 0} \eta_{P}^{A} & =\lim _{P_{u} \rightarrow 0} \frac{1}{P_{u}} \sum_{k=1}^{K} E\left\{\log _{2}\left(1+\frac{P_{u}\left|g_{n n k}^{H} f_{P, n k}^{A}\right|^{2}}{P_{u} \sum_{i=1}^{K}\left|g_{n n k}^{H} f_{P, n i}^{A}\right|^{2}+P_{u} \sum_{l=1}^{L} \sum_{i=1}^{K}\left|g_{\ln k}^{H} f_{P, l i}^{A}\right|^{2}+1}\right)\right\} \\
& =\sum_{k=1}^{K} E\left\{\left|g_{n n k}^{H} f_{I P, n k}^{A}\right|^{2}\right\} \\
\lim _{P_{u} \rightarrow 0} \eta_{I P}^{A} & =\lim _{P_{u} \rightarrow 0} \frac{(T-\tau)}{T P_{u}} \sum_{k=1}^{K} E\left\{\log _{2}\left(1+\frac{\left|g_{n n k}^{H} f_{I P, n k}^{A}\right|^{2}}{\sum_{i=1}^{K}\left|g_{n n k}^{H} f_{I P, n i}^{A}\right|^{2}+\sum_{l=1}^{L} \sum_{i=1}^{K}\left|g_{\ln k}^{H} f_{I P, l i}^{A}\right|^{2}+\frac{1}{P_{u}}}\right)\right\} \\
& =\frac{(T-\tau)}{T} \sum_{k=1}^{K} E\left\{\left|g_{n n k}^{H} f_{I P, n k}^{A}\right|^{2}\right\}
\end{aligned}
$$

When $P_{u} \rightarrow \infty$ :

$$
\begin{aligned}
\lim _{P_{u} \rightarrow \infty} \eta_{P}^{A} & =\lim _{P_{u} \rightarrow \infty} \frac{1}{P_{u}} \sum_{k=1}^{K} E\left\{\log _{2}\left(1+\frac{P_{u}\left|g_{n n k}^{H} f_{P, n k}^{A}\right|^{2}}{P_{u} \sum_{i=1}^{K}\left|g_{n n k}^{H} f_{P, n i}^{A}\right|^{2}+P_{u} \sum_{l=1}^{L} \sum_{i=1}^{K}\left|g_{\ln k}^{H} f_{P, l i}^{A}\right|^{2}+1}\right)\right\} \\
& =0 \\
\lim _{P_{u} \rightarrow \infty} \eta_{I P}^{A} & =\lim _{P_{u} \rightarrow \infty} \frac{(T-\tau)}{T P_{u}} \sum_{k=1}^{K} E\left\{\log _{2}\left(1+\frac{\left|g_{n n k}^{H} f_{I P, n k}^{A}\right|^{2}}{\sum_{i=1}^{K}\left|g_{n n k}^{H} f_{I P, n i}^{A}\right|^{2}+\sum_{l=1}^{L} \sum_{i=1}^{K}\left|g_{\ln k}^{H} f_{I P, l i}^{A}\right|^{2}+\frac{1}{P_{u}}}\right)\right\} \\
& =0
\end{aligned}
$$

\section{Results}

A simulation environment was built, cell number $L=4$, cell radius $r=1000 \mathrm{~m}$, users in each cell $K=10$, and users were distributed around the base station in the radius $r_{h}=1000 \mathrm{~m}$. Assume the distance from the $k$ user to the base station is $r_{k}$, 
$\beta_{k}=z_{k} /\left(r_{k} / r_{h}\right)^{v}$ is the macro fading of user $k$ to the base station, the shadow fading coefficient is $z_{k}=8 d B$, path loss coefficient $v=3.8$.

Figure 5 shows relation of the cell spectral efficiency and number of cell antennas $M$ in the condition of known CSI of multi cell MIMO system. The system parameters are: cell number $L=3$, cell CSI is known, the number of cell antennas $M$ are changed from 20 to 500, the transmission power of base station is $P=E / M, E=[20,30] d B$.

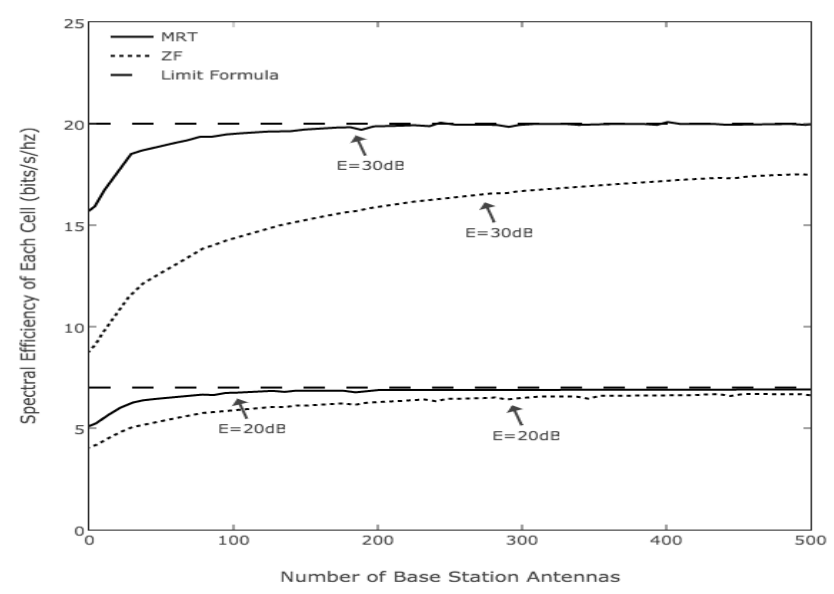

Figure 5. The Spectral Efficiency of Multi Cell System with known CSI

The simulation results in figure 5 indicate that when the CSI of a multi cell MINO system is known, and the base station transmit power is reduced to $1 / M$ single antenna, with the increase of $M$, the cell spectral efficiency of MRT and ZF tends to a limit value, and the system performance is equivalent to the single cell system.

Figure 6 shows the relationship of spectral efficiency and number of cell antennas in the multi and single cell environment with known CSI. The system parameters are: cell CSI is known, the number of cell antennas $M$ are changed from 20 to 500, the transmission power of base station is $P=E / M, E=30 \mathrm{~dB}$.

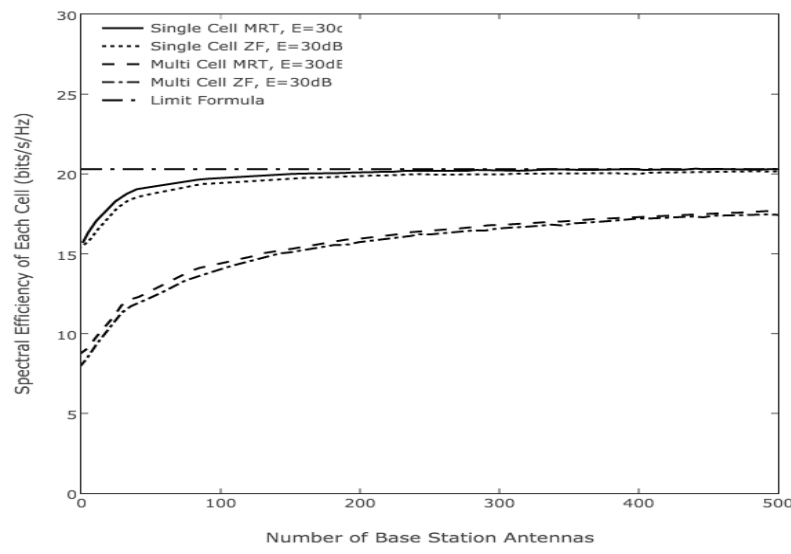

Figure 6. The Spectral Efficiency of Multi and Single Cell System with known CSI

The simulation results in Figure 6 indicate that with the increase of the number of antennas, the spectral efficiency of the multi cell system is close to that of the single cell 
system. When $M=500$, the difference of spectrum efficiency is only $0.1 \mathrm{bits} / \mathrm{s} / \mathrm{Hz}$ in the two systems. It shows that the increase of the antennas number in base station is effective in eliminating the inter-cell interference in multi cell systems.

As in single cell, Figure 7 shows the relationship of cell antennas number and the spectral efficiency with decrease of transmits power to $1 / M$ and limit CSI in the multi cell MIMO system. The system parameters are: cell number $C=3$, cell CSI is known, the number of cell antennas $M$ are changed from 20 to 500, the transmission power of base station is $P=E / M, E=30 d B$.

The simulation results in Figure 7 indicate that the cell spectral efficiency is increased with the increase of user transmits pilot, and gradually tends to the limit with the increase of the number of antennas. At the same time, it shows the performance gap between ZF pre-encoding and MRT pre-encoding, ZF method has a performance advantage comparing to MRT.

\section{Conclusions}

With the interference and spectral efficiency model, we analysis the performance of MIMO system in a simulation environment. The empirical study mainly includes the asymptotic spectrum efficiency of large scale MIMO system in multi cell systems, MRT and ZF pre-encoding's limit performance. When the transmission power is reduced to $1 / M$ of a single antenna base station, with the increase of $M$, though the simulation analysis, we have the conclusions as follows:

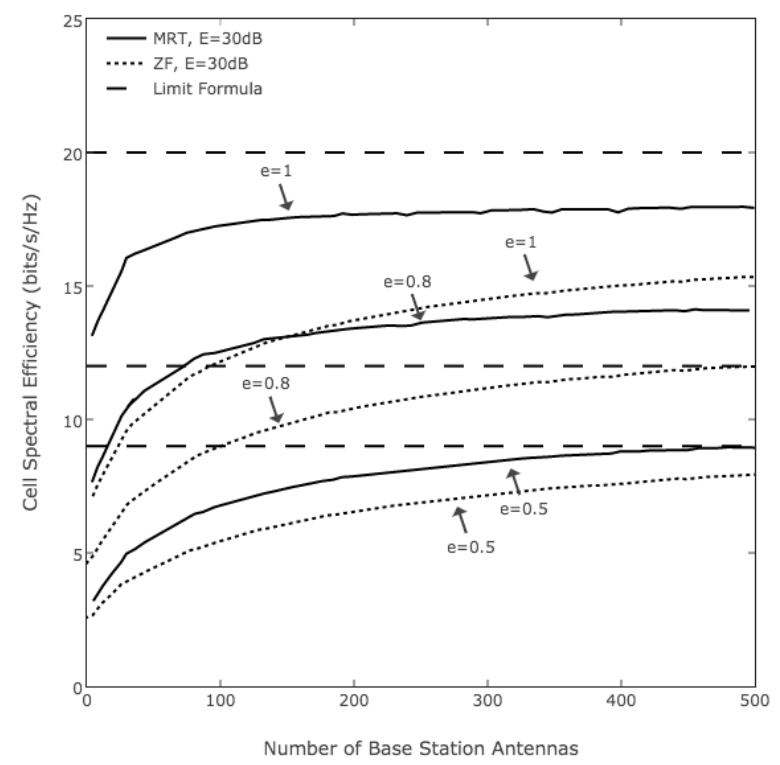

\section{Figure 7. The Spectral Efficiency of Multi and Single Cell System with Finite} CSI

(1) Simple prevention can eliminate the interference in a cell or between cells with known CSI and the base station spectral approximate to a constant.

(2) When the base station is known to have finite CSI, the multi cell system is affected by the user pilot pollution, and the inter cell interference cannot be eliminated completely, the spectrum efficiency of the system tends to a certain value with the increase of user transmits pilot. The value is related to the user sends uplink pilot sequence and channel slow fading, and the spectrum efficiency of the system is proportional to the pilot power transmitted by the user. However, when the base station transmits power decreased to $1 / M$ of a single antenna base station, the system is limited by interference. 
(3) The ZF pre-encoding is equal to the MRT pre-encoding under limited conditions.

Large scale MIMO technology uses the random approximation of the space channel, and the fast fading and additive noise is mitigated by the simple pre-treatment or detection method. Large scale antenna array can not only improve the system spectrum efficiency and reduce the transmission power, but also simplify the processing operation of the base station, reducing the complexity of the system. The MIMO system can satisfy the requirements of high speed and low complexity in the next generation mobile communication systems.

The limitation of this study exists in the following aspects. This paper is carried out under ideal conditions, assuming there is no connection within the base station's large antenna array, this is not possible in the actual system. And in the condition of limited antenna number and transmission power, the time to achieve the peak speed with certain number of users is worth further study.

\section{Acknowledgements}

This work is supported by Scientific and Technological Research Program of Chongqing Municipal Education Commission (Grant No. KJ132206).

\section{References}

[1] P. Pattanayak and P. A. Kumar, "Computationally efficient genetic algorithm for MIMO broadcast scheduling”, Applied Soft Computing, vol. 37, (2015), pp. 545-553.

[2] S. Granlund, L. Liu, C. Zhang, and V. Öwall, "A low-latency high-throughput soft-output signal detector for spatial multiplexing MIMO systems", Microprocessors and Microsystems, vol. 39, (2015), pp. 901-908.

[3] H. He, M. Tian, Z. Wang, W. Zhang, and X. Su, "Efficient user selection for multi-cell multi-user MIMO systems with limited backhaul", The Journal of China Universities of Posts and Telecommunications, vol. 19, (2012), pp. 18-23.

[4] B. Hu, Y. Liu, G. Xie, J. Gao and Y. Yang, "Energy efficiency of massive MIMO wireless communication systems with antenna selection" The Journal of China Universities of Posts and Telecommunications, vol. 21, (2014), pp. 1-8.

[5] S. Chao, B. Chen and C. Li, "Grid cell based detection strategy for MIMO radar with widely separated subarrays", AEU - International Journal of Electronics and Communications, vol. 66, (2012), pp. 741751.

[6] Y. Zhang, C. Gu, R. Shu, Z. Zhou and W. Zou, "Interference Alignment in Multi-user MIMO Systems", ICT Express, vol. 1, (2015), pp. 5-9.

[7] J. Jiang, W. Lv, B. Sun, G. Lu and Y Xie, "Interference alignment scheme for massive MIMO system", The Journal of China Universities of Posts and Telecommunications, vol. 21, (2014), pp. 19-24.

[8] V. C. Tran, M. T. Le, X. N. Tran and T. Q. Duong, "MIMO cooperative communication network design with relay selection and CSI feedback", AEU - International Journal of Electronics and Communications, vol. 69, (2015), pp. 1018-1024.

[9] P. Patcharamaneepakorn, S. Armour and A. Doufexi, "Modified leakage-based transmit filter designs for multi-user MIMO systems", Physical Communication 13, Part C, (2014), pp. 73-87.

[10] M. Baadeche and F. Soltani, "Performance analysis of ordered CFAR detectors for MIMO radars", Digital Signal Processing, vol. 44, (2015), pp. 47-57.

[11] P. Nagaradjane, Y. A. Rajan, P. Muralidharan and S. K. V. Sarathy, "Performance of multi-user transmitter preprocessing assisted MIMO system over correlated frequency-selective channels", Physical Communication, vol. 7, (2013), pp. 61-72.

[12] S. J. Lu, R. Y. Chang, W. H. Chung and C. E. Chen, "Realizing high-accuracy transmission in high-rate data broadcasting networks with heterogeneous users via cooperative communication”, Digital Signal Processing, vol. 25, (2014), pp. 93-103.

[13] W. Peng, and F. Adachi, "Single-carrier frequency domain adaptive antenna array for uplink multi-user MIMO transmission in a cellular system", Physical Communication, vol. 8, (2013), pp. 22-30.

[14] M. Z. Shakir, H. Tabassum, K. A. Qaraqe, E. Serpedin and M. S. Alouini, "Spectral and energy efficiency analysis of uplink heterogeneous networks with small-cells on edge", Physical Communication, vol. 13, Part B, (2014), pp. 27-41.

[15] E. Biglieri, "MIMO wireless communications", Cambridge university press, (2007).

[16] H. Huang, C. B. Papadias and S. Venkatesan, "MIMO Communication for Cellular Networks", Springer Science \& Business Media, (2011). 
[17] A. B. Gershman and N. D. Sidiropoulos, "Space-time processing for MIMO communications" John Wiley, (2005).

\section{Authors}

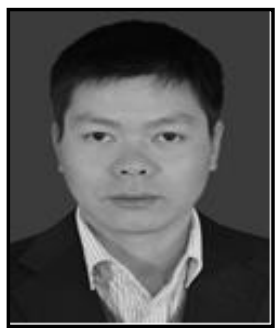

Zhiyong Renb, he was born in 1971. He received his M.S. degree in Electric circuit and systematic specialty from ChongQing University in 2007. Now, $\mathrm{He}$ is a vice professor in ChongQing College of Electronic Engineering. His academic papers has been published more than 10. His main research interests include signal processing and communication technology. 
International Journal of Future Generation Communication and Networking Vol. 9, No. 6 (2016) 